\title{
Importance of biomarkers in glioblastomas patients receiving local BCNU wafer chemotherapy
}

\author{
Steffi Urbschat ${ }^{1}$, Christoph Sippl ${ }^{1}$, Jana Engelhardt ${ }^{1}$, Kai Kammers ${ }^{2}$, Joachim Oertel ${ }^{1}$ and Ralf Ketter ${ }^{1 *}$ (D
}

\begin{abstract}
Background: To assess the influence of molecular markers with potential prognostic value to groups of patients with newly diagnosed glioblastoma patients were examined: group A with 36 patients (surgical resection plus standard combined chemoradiotherapy) and group B with 36 patients (surgical resection, standard combined chemoradiotherapy plus carmustine wafer implantation).

Our aim was to determine chromosomal alterations, methylation status of MGMT, p15, and p16 (CDKN2A) in order to analyse the influence on patient survival time as well as radio- and chemotherapy responses. Promoter hypermethylation of MGMT, p16, and p15 genes were determined by MS-PCR. Comparative genomic hybridisation $(\mathrm{CGH})$ analyses were performed with isolated, labelled DNA of each tumor to detect genetic alterations.
\end{abstract}

Results: Age of onset of the disease showed a significant effect on overall survival (OS) $(p<0.0001)$. Additional treatment with carmustine wafer (group B) compared to the control group (group A) did not result in improved OS $(p=0.562)$. Patients with a methylated MGMT promotor showed a significant longer OS compared to those patients with unmethylated MGMT promotor $(p=0.041)$. Subgroup analyses revealed that patients with methylated p15 showed a significant shorter OS when administered to group B rather than in group A $(p=0.0332)$. In patients additionally treated with carmustine wafer an amplification of $4 q 12$ showed a significant impact on a reduced OS $(p=0.00835)$. In group $B$, a loss of $13 q$ was significantly associated with a longer OS $(p=0.0364)$. If a loss of chromosome 10 occurred, patients in group B showed a significantly longer OS ( $p=0.0123)$.

Conclusion: A clinical benefit for the widespread use of additional carmustine wafer implantation could not be found. However, carmustine wafer implantation shows a significantly improved overall survival if parts of chromosome 10 or chromosome 13 are deleted. In cases of $4 q 12$ amplification and in cases of a methylated p15 promotor, the use of carmustine wafers is especially not recommended.

The MGMT promoter methylation is a strong prognostic Biomarker for benefit from temozolomide and BCNU chemotherapy.

Keywords: Glioblastoma, CGH, Carmustin wafer, Prognostic factors, Standard combined radiochemotherapy

\section{Background}

Glioblastoma multiforme (GBM) is the most aggressive and most common form of primary brain cancer [1]. At an incidence of 3 to 4 in 100,000 and a slight male predominance of 1.2-1.9:1 it can strike all ages [2-4]. The

\footnotetext{
* Correspondence: ralf.ketter@uks.eu

${ }^{1}$ Department of Neurosurgery, Saarland University, 66421 Homburg/Saar, Germany

Full list of author information is available at the end of the article
}

gold standard treatment for GBM is a grand total resection combined with radiochemotherapy consisting of 60 Gy radiation and temozolomide (TMZ) according to Stupp et al. [5]. Another option in the treatment of GBM is, in addition to the Stupp-regime, the implantation of carmustin (BCNU) eluted wafer into the resection cave. Hence a higher concentration of antineoplastic agents can be released to the tissue adjacent to the tumor. This strategy minimizes the systemic effects and maximizes the anti-neoplastic effect by bypassing the blood brain barrier [6]. Despite this theoretical 
Table 1 Primer for MS-PCR

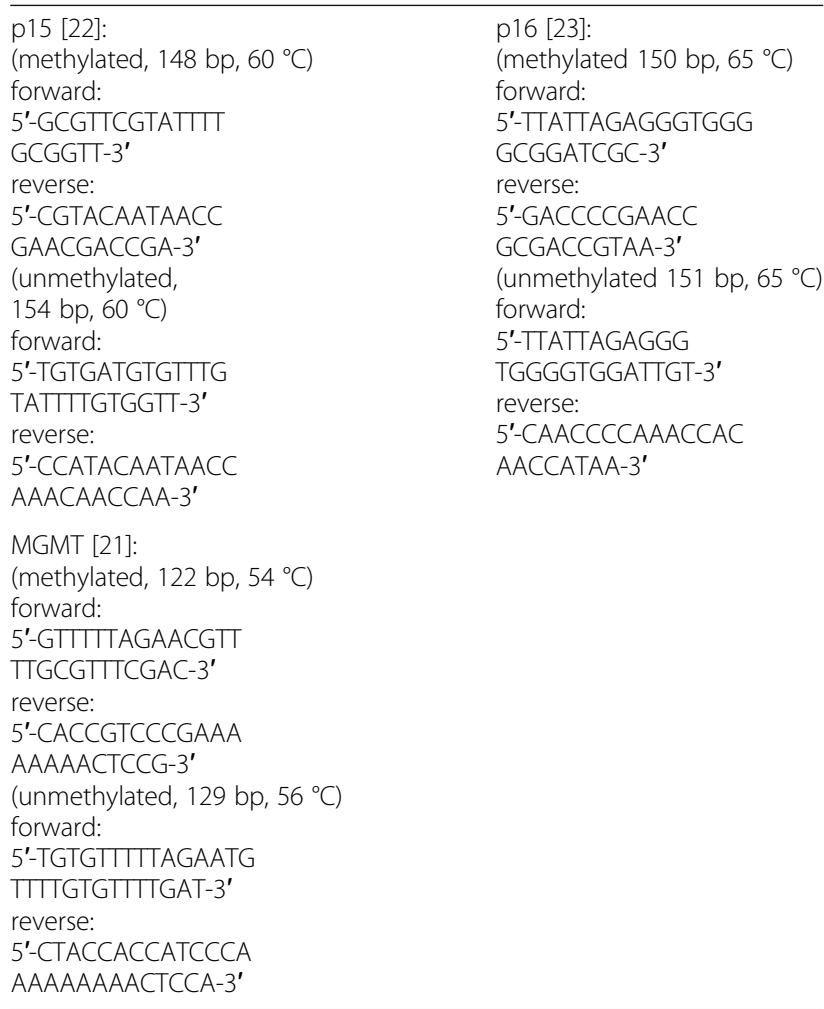

advantage, the clinical benefit remains in dispute. Some authors showed a benefit regarding overall survival (OS) when carmustin wafers were implanted postresectionally [7, 8]. In contrast Pallud et al. could not show a long-term benefit in (OS) at a cohort of 354 patients [9]. However, some adverse effects like cerebral edema and postoperative wound infection can be increased [10]. Therefore, it would be eligible to predict the response characteristics of a carmustin wafer therapy. Whether carmustine wafer implantation is recommendable or not could depend on patients' individual (genetic) characteristics.

A marker, which is known to predict chemotherapy response in GBM, is the promotor methylation status of O6-methyl-guanine-methyl transferase (MGMT), a DNA repair enzyme. This protein repairs alkylating DNA damage induced by TMZ in tumor cells and hereby promotes tumor progression of GBM [11]. Several clinical studies showed that low MGMT expression was significantly related to ameliorated TMZ therapy response [12-19]. Carmustine as the active drug in carmustine wafer is also a DNA alkylating agent, which can be counteracted by MGMT [20]. Another potential marker for the clinical course of GBM is a hypermethylation status of p15 that was shown to be associated with a shorter OS [21].

Furthermore, chromosomal alterations like gains on chromosome 7 and losses of parts of chromosome 10 and of/parts of the short arm of chromosome 9 are common phenomenon in glial tumors. Especially losses on chromosome $9 \mathrm{p}$ and 10q are often associated with a poor prognosis for patients with GBM [22]. Losses of chromosome 9 affects p16, a cell cycle controlling protein located on 9p21.3.

The aim of this experimental trial was to find new markers for treatment response in GBM on genetic and also epigenetic levels and to investigate the effects of additional carmustine wafer therapy on known markers. In particular, we focused on genes regulating cell cycle, e.g., p15, p16, and the DNA repair enzyme MGMT. For further stratification and subgroup analyses, we also incorporated two different treatment modalities: one patient cohort treated according to Stupp et al. and the other patient cohort received carmustine wafer implantation after resection in addition the Stupp et al. regime [5].

\section{Methods}

\section{Patients}

In this trial, we enclosed 72 patients with newly diagnosed GBM who underwent surgery between 2005 and

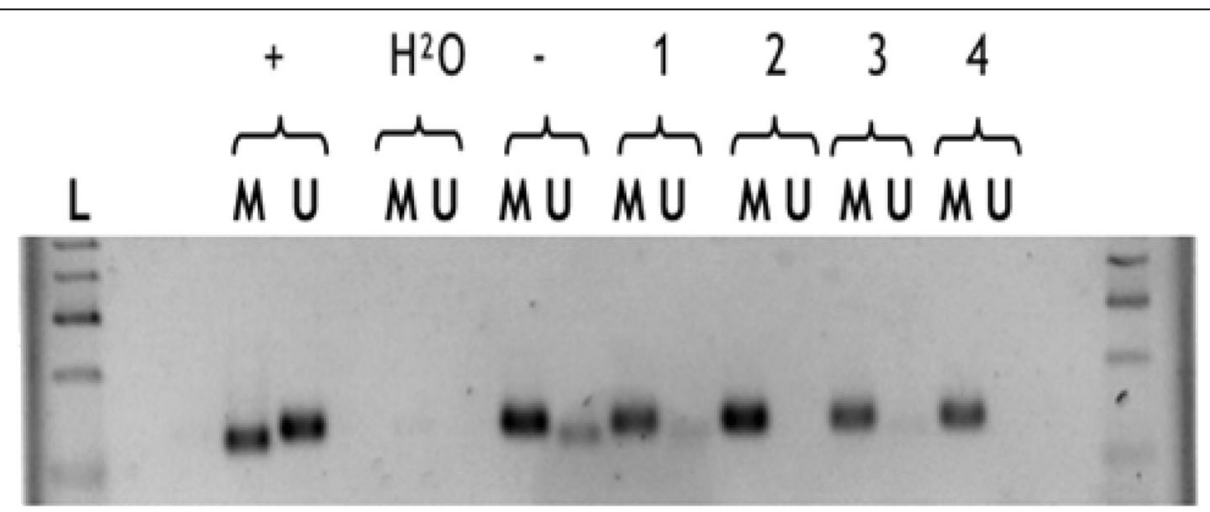

Fig. 1 Methylation specific PCR of MGMT promotor in GBMs. L= Molecular size marker; $U=$ unmethylated DNA; $M=$ methylated DNA; + = positive control; - = negative control; $\mathrm{H}_{2} \mathrm{O}=$ blank value; 1 = case 1442/08; 2 = case 1510/10; 3 = case 1219/19; 4 = case 288/08 
Table 2 Clinical, epigenetic and CGH results (univariates Coxmodel)

\begin{tabular}{|c|c|c|c|c|c|}
\hline & $\mathrm{HR}$ & $1 / \mathrm{HR}$ & lover.95 & upper.95 & $p$ value \\
\hline \multicolumn{6}{|c|}{ Clinical data } \\
\hline groupe & 1.1561 & 0.8649 & 0.7079 & 1.8880 & $p=0.5620$ \\
\hline age & 1.0479 & 0.9542 & 1.0242 & 1.0721 & $p=0.6081$ \\
\hline gender & 1.1889 & 0.8410 & 0.7151 & 1.9767 & $p=0.5046$ \\
\hline \multicolumn{6}{|c|}{ Methylation } \\
\hline MGMT & 0.5929 & 1.6866 & 0.3590 & 0.9789 & $p=0.041$ \\
\hline p15 & 0.7883 & 1.2684 & 0.4065 & 1.5287 & $p=0.4816$ \\
\hline p16 & 1.5724 & 0.6359 & 0.6735 & 3.6711 & $p=0.2954$ \\
\hline \multicolumn{6}{|c|}{ Comparative genetic hybridization [CGH) } \\
\hline $1 q$ & 2.1684 & 0.4611 & 0.6683 & 7.0355 & $p=0.1974$ \\
\hline amp4q12 & 2.3050 & 0.4338 & 1.1815 & 4.4965 & $p=0.01431$ \\
\hline 7 & 1.5579 & 0.6418 & 0.7591 & 3.1973 & $p=0.2267$ \\
\hline amp7p12 & 1.4766 & 0.6771 & 0.8132 & 2.6813 & $p=0.2002$ \\
\hline $9 p$ & 1.0812 & 0.9248 & 0.6633 & 1.7626 & $p=0.7538$ \\
\hline 10 & 1.0692 & 0.9352 & 0.6536 & 1.7490 & $p=0.7897$ \\
\hline $10 q$ & 0.8369 & 1.1948 & 0.4945 & 1.4162 & $p=0.5071$ \\
\hline $12 q$ & 1.0097 & 0.9903 & 0.5659 & 1.8015 & $p=0.9737$ \\
\hline 13 & 0.6853 & 1.4591 & 0.4169 & 1.1264 & $p=0.1361$ \\
\hline 17 & 1.0081 & 0.9919 & 0.5704 & 1.7816 & $p=0.9778$ \\
\hline 20 & 1.4714 & 0.6796 & 0.8380 & 2.5835 & $p=0.1787$ \\
\hline 22 & 1.0570 & 0.9459 & 0.5908 & 1.8912 & $p=0.8515$ \\
\hline
\end{tabular}

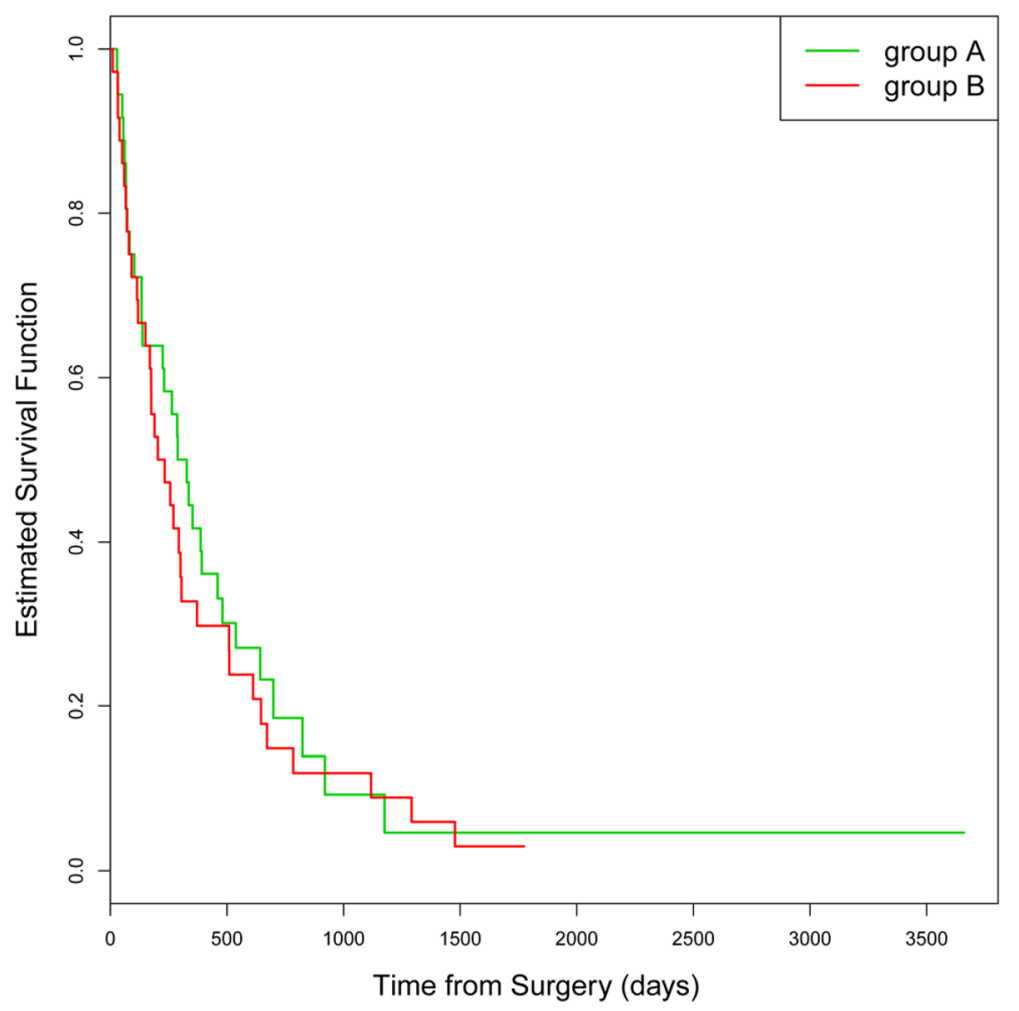

Fig. 2 OS in group A (standard Stupp regime) and group B (standard Stupp regime + carmustine wafer) 


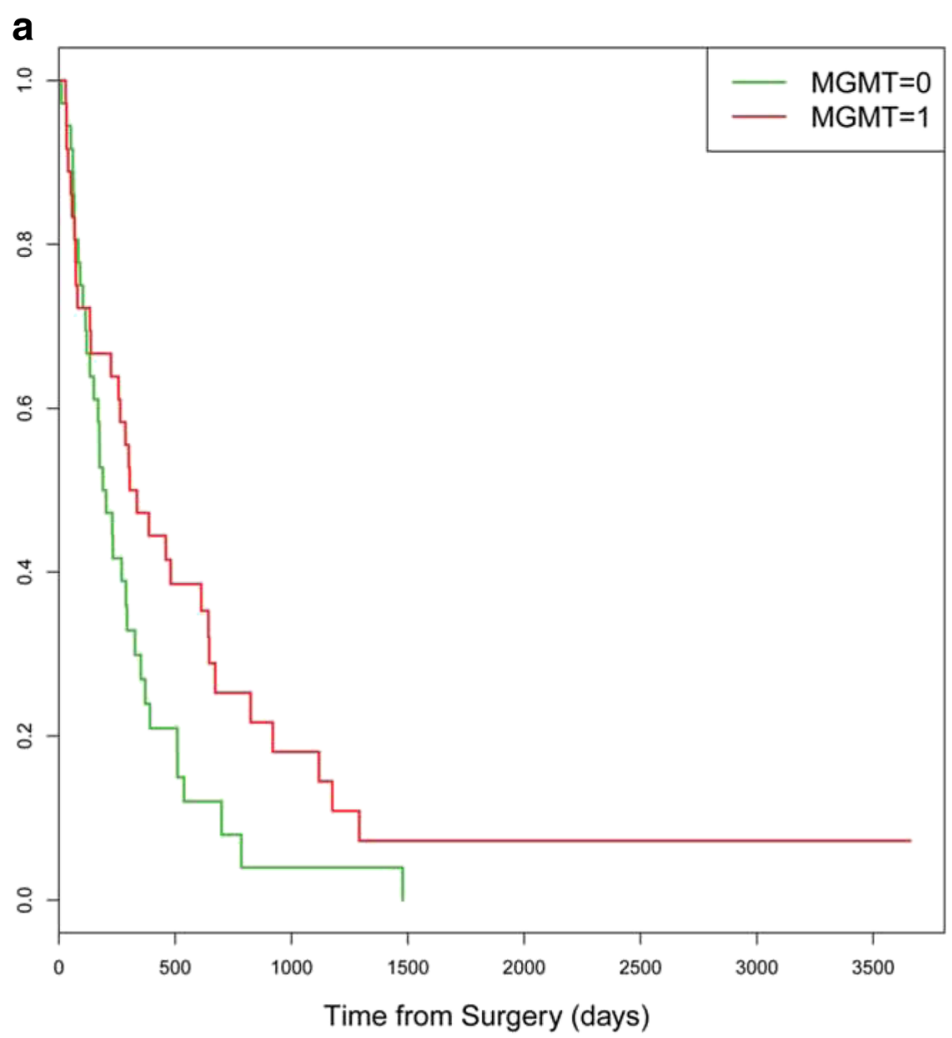

b
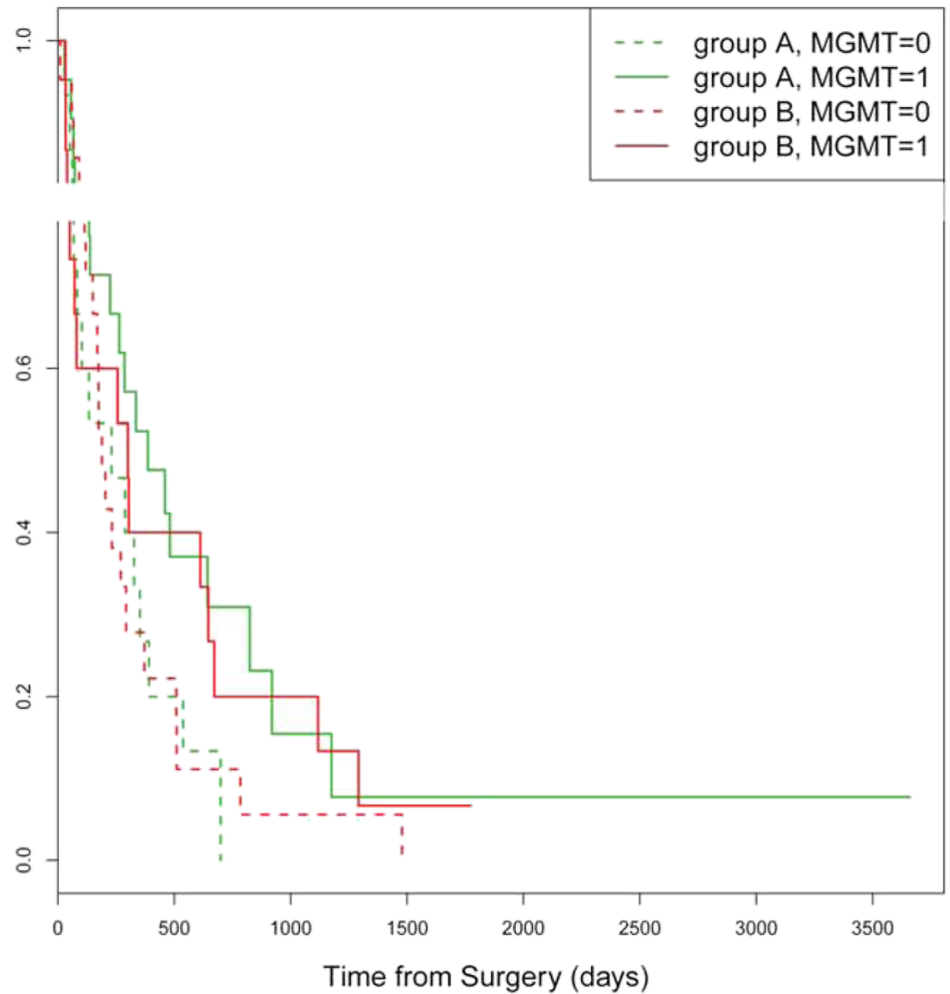

Fig. 3 a OS depending on MGMT methylation status. Green: no methylation of MGMT promoter. Red: methylation of MGMT promoter. b: OS in group A and B depending on MGMT methylation status. 0: no methylation of MGMT promoter. 1: methylation of MGMT promoter 


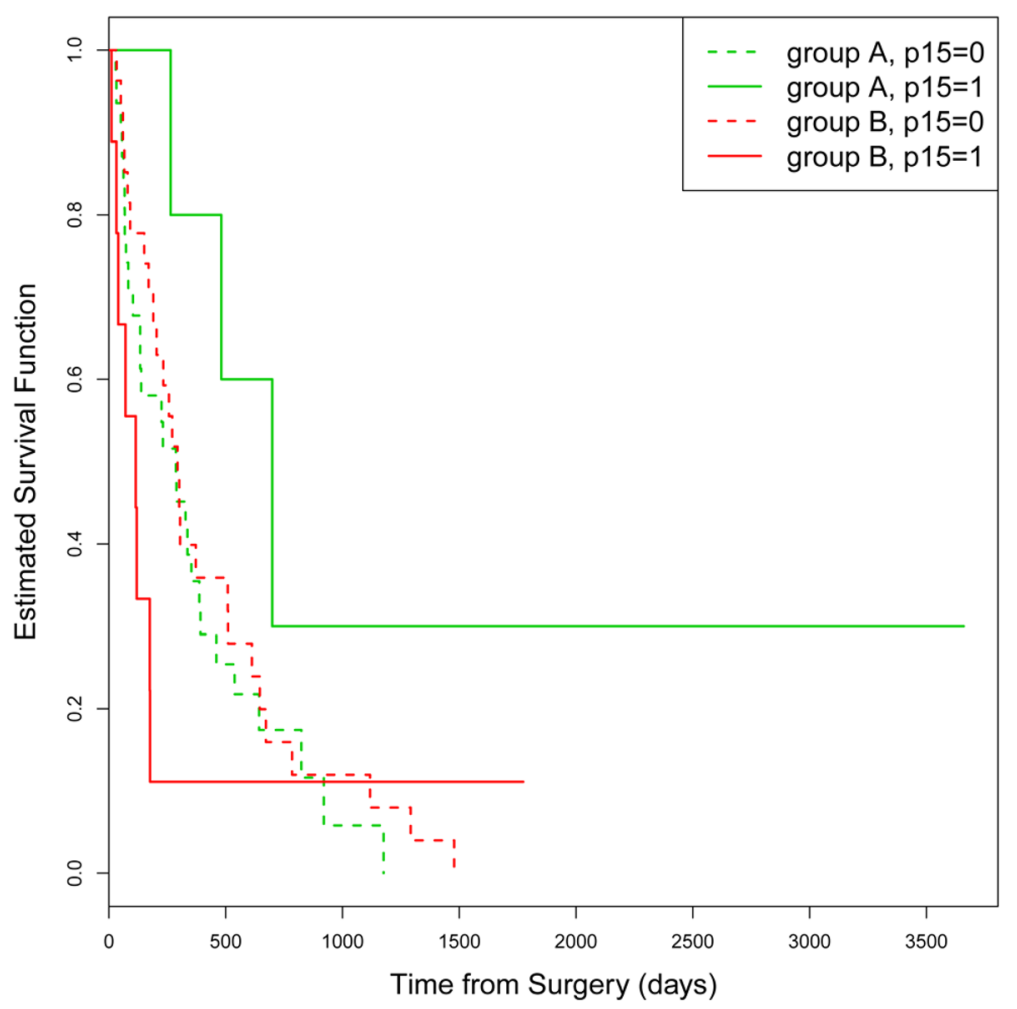

Fig. 4 OS in group A and B depending on p15 methylation status. Green: group A (Stupp regime). Red: group B (Stupp regime + carmustine wafer)

2012 at the department of neurosurgery at the university medical center Homburg/Saar, divided into two matched pair groups with 36 patients each. After tumor resection one group was treated according to the standard Stupp regime (group A), whereas the other cohort was treated according to the standard Stupp regime with carmustine wafer implantation (group B).

Tumor tissue was collected at the time of surgery, if clinically indicated. All tissue samples were frozen immediately after the tumor was resected using liquid nitrogen and stored in our tumor bank at $-80{ }^{\circ} \mathrm{C}$. This study was approved by the local ethic board of Saarland and written informed consent was obtained from all patients.

\section{Methylation analysis}

DNA isolation was performed using DNA isolation kit (Qiagen, QIAamp DNA Mini Kit 50). The methylation status of promoter regions of the genes p15, p16 and MGMT were determined by methylation specific polymerase chain reaction (MS-PCR). Therefore, 500 ng DNA of each tumor specimen, as well as appropriate control samples were bisulfite-treated (ZYMO RESEARCH, EZ DNA Methylation-Gold Kit 200) [23]. In summary, thus unmethylated cytosine is converted to uracil, whereas methylated cytosine remained unchanged. The modified DNA was recovered by ethanol precipitation and dissolved in water. For the analysis of the methylation status the primer sequences listed in Table 1 were used.

PCR was performed with a $25 \mu$ l reaction volume and 38 PCR cycles. All PCR products were separated by electrophoresis on a $2 \%$ agarose gel. As methylated and unmethylated control we used Universal Methylated $\mathrm{Hu}$ man DNA (ZYMO RESEARCH). As blank value we added water in place of DNA [Fig. 1].

\section{CGH analysis}

Comparative genomic hybridisation $(\mathrm{CGH})$ was used to screen the tumors for chromosomal imbalances. Metaphasic preparation was acquired via short term lymphocytic culture. CGH was performed as described previously [24].

\section{Statistics}

Comparisons of survival times between groups defined by clinical variables, methylation status and parts of chromosome deletions were performed by Kaplan-Meier curves and with two-sided log rank tests. Methylation index was defined as percentage of patients with promotor methylation of the total cohort in percent. Univariate Cox regression [25] analysis was performed to identify significant predictors for overall survival (OS). Effects of individual predictors on $\mathrm{OS}$ in all models were 

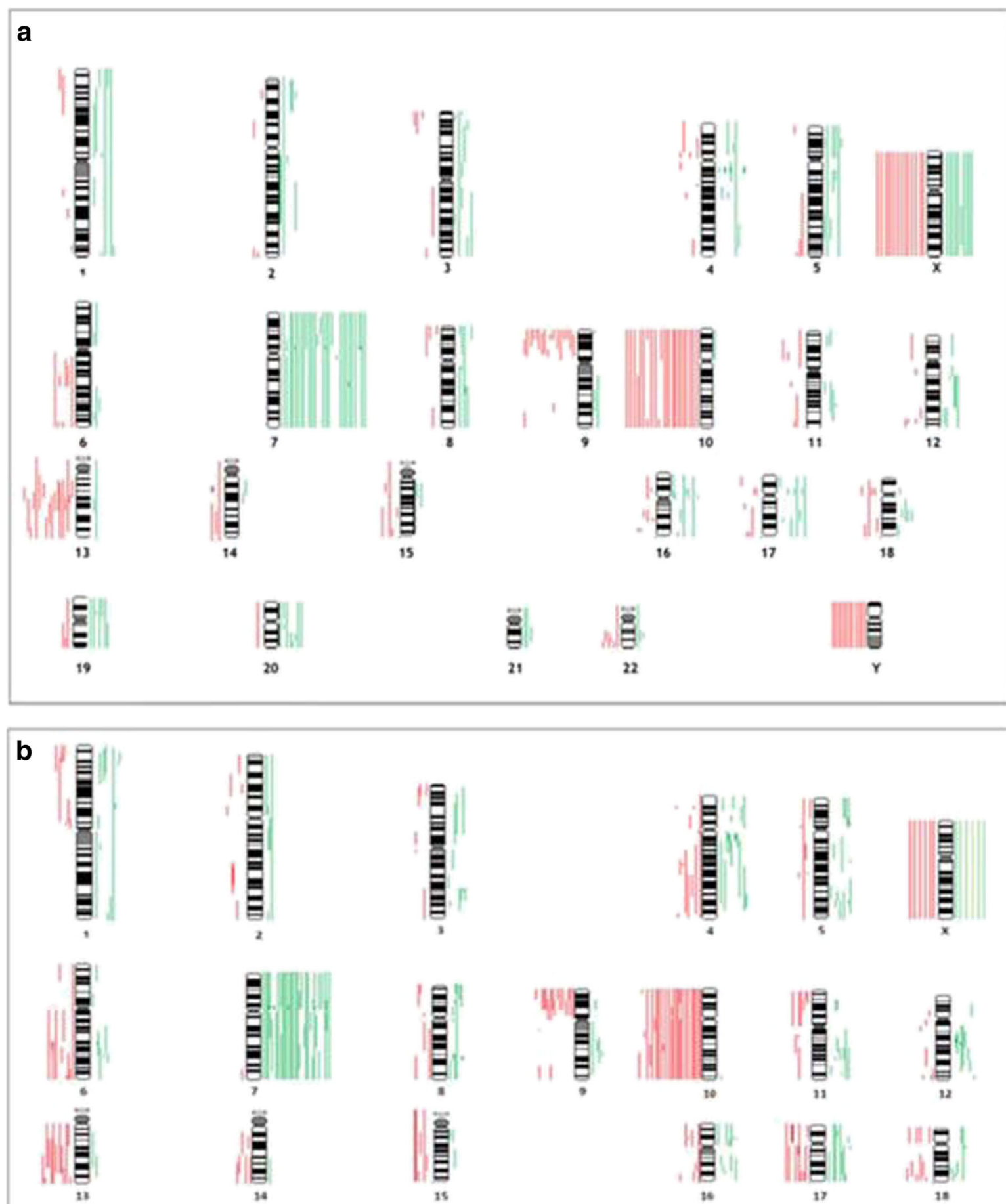

|| 晋
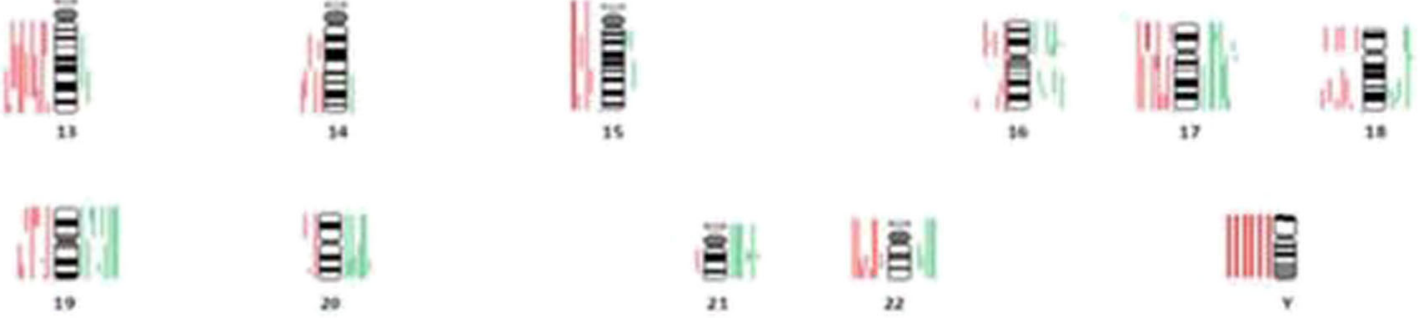

Fig. 5 a Overview of genetic imbalances of the Carmustin-group. Lines on the left represent losses, and lines on the right represent gains; amplifications are in bold. $\mathbf{b}$ : Overview of genetic imbalances of the conventionally treated-group. Lines on the left represent losses, and lines on the right represent gains; amplifications are in bold 
Table 3 Clinical chacteristics and CGH results of the Carmustin group

\begin{tabular}{|c|c|c|c|}
\hline Cases & Histology & $\begin{array}{l}\text { Ages/ } \\
\text { gender }\end{array}$ & CGH - results \\
\hline $\begin{array}{l}2065 / \\
08\end{array}$ & pGBM & $80 / f$ & $\begin{array}{l}+X,+7,-9 p,-10,-14 q 24 q t e r,-17 p 12 \text { pter, } \\
-22 q 11.2 q 13.2\end{array}$ \\
\hline $\begin{array}{l}270 / \\
10\end{array}$ & pGBM & $80 / m$ & $\begin{array}{l}-4,-6,+7,-8 q 21.1 q \text { ter, }+9 q 12 q 34.1,-10, \\
-11 p 14 p t e r,-16,-17 q,-18 p 11.2 p t e r,+19, \\
+20,+21\end{array}$ \\
\hline
\end{tabular}

$\begin{array}{ll}\text { 1526/ pGBM 75/m } \quad & -X_{1}+1 p 33 p t e r,-1 p 11 p 22.1, \text { amp4q12, } \\ 07 & +6 p 12 p 21.3,+7,-9 p 13 p t e r,-10, \\ & +11 q 23.3 q 25,+15 q 11.2 q 14,+16 p\end{array}$

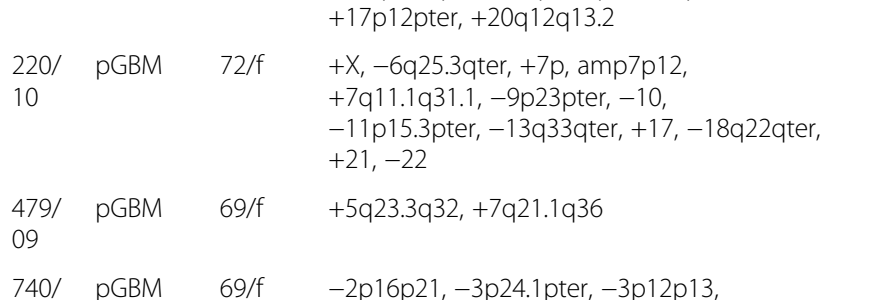

Table 3 Clinical chacteristics and CGH results of the Carmustin group (Continued)

\begin{tabular}{|c|c|c|c|}
\hline $\begin{array}{l}1809 / \\
11\end{array}$ & pGBM & $55 / \mathrm{m}$ & $\begin{array}{l}\text {-1p26.1pter, +3p12p22, amp4q12, } \\
+5 p 14 p t e r,+5 q 11.2 q 21, \\
\text {-6p22.3pter, +6q22.2qter, }+7,+8 p 11.2 p 21.1, \\
+8 q 11.2,+8 q 22.3 q 23,-9 p,+9 q 21.3 q 22.3, \\
-9 q 34.1 q t e r,-10 p 11.1 p 12.3,-10 q \\
+11 p 14 p 15.3,+11 q 13.2 q 14.3,+12 q 12 q 23, \\
-13 q 11 q 32, \\
-14 q 22 q t e r,-15\end{array}$ \\
\hline $\begin{array}{l}1362 / \\
07\end{array}$ & pGBM & $52 / \mathrm{m}$ & $\begin{array}{l}-1 p 34.3 p t e r,-5 q 12 q 14,+7,-9 p 11 p 22,-10 \\
+18 q 21.3 q \text { ter, }+19 p\end{array}$ \\
\hline $\begin{array}{l}288 / \\
08\end{array}$ & pGBM & $52 / \mathrm{m}$ & $\begin{array}{l}\text { amp7p12, }+7 q 31.3 q 35,+9 q 21.2 q 32,-10, \\
-11 q 12 q 13.2, \\
-13 q 12.3 q 31,-17 q 11.1 q 21.1\end{array}$ \\
\hline $\begin{array}{l}1350 / \\
06\end{array}$ & sGBM & $46 / \mathrm{m}$ & $\begin{array}{l}+1 q 12 q 23, \text { amp4q12, +4q12q22, +8q23qter, } \\
-1 p 21 p 36.1, \\
-4 q 28 q t e r,-6 q,-13 q 14.3 q 33,-18 q 12.3 q 23, \\
-19 q 13.1 q 13.3 \\
-22 q 12.3 q \text { ter }\end{array}$ \\
\hline $\begin{array}{l}1455 / \\
08\end{array}$ & pGBM & $46 / \mathrm{m}$ & $\begin{array}{l}+4 p 14 p 15.1, \text { amp4q12, +7p, +8p21.2p22, } \\
+11 q 14.1 q 22.3\end{array}$ \\
\hline $\begin{array}{l}768 / \\
09\end{array}$ & pGBM & $44 / \mathrm{m}$ & $\begin{array}{l}-6 q 25.1 q \text { ter, }+7 q 21.2 q 32,+12 q 13.2 q 21.1 \\
-18 p,-19\end{array}$ \\
\hline P.E. & pGBM & $44 / m$ & $\begin{array}{l}+1 q 43 q t e r,+2 p 11.1 p 13,+2 q 13 q 22, \\
+2 q 37.1 q t e r,+3 p 13 p 33,+4 p 15.3 p t e r \\
+5 p 14 p t e r,+5 q 31.1 q t e r,+6 q 23.1 q 22.3,+7, \\
+9 q 22.3 q 31,-10 q 22.1 q 22.3,+11 q 23.3 \\
+12 q 15 q 21.3,-13,+16 q 23 q 24,+17 q 12 q 24 \\
+18 q 22\end{array}$ \\
\hline $\begin{array}{l}1741 / \\
08\end{array}$ & $\mathrm{pGBM}$ & $54 / f$ & $-9 p 21$ pter, $-10,-17 q,+18 q 12.3 q 22$ \\
\hline $\begin{array}{l}1701 / \\
07\end{array}$ & pGBM & $67 / m$ & $\begin{array}{l}\text { amp 4q12, } 7,+17 q,-4 q 21.3 q 34,-5,-10, \\
-11 q 12 q 21 \\
-13 q 14.1 \text { ter, }-17 p,-19 p 11 \text { pter }\end{array}$ \\
\hline $\begin{array}{l}1935 / \\
08\end{array}$ & pGBM & $70 / \mathrm{m}$ & $\begin{array}{l}\text { amp4q12, +7q11q21.3, -9p21 pter, }-10, \\
-18 q 22 q \text { ter, }-20 p 11.2 \text { pter }\end{array}$ \\
\hline $\begin{array}{l}1618 / \\
09\end{array}$ & pGBM & $54 / f$ & $\begin{array}{l}-2 q 24.1 q 34,-3 p 11.1 p 14.1,-3 q 11.1 q 13.1, \\
-4 q 24 q 26,-5 p 11 p 12, \\
-6 p 11 p 12, \text { amp7p12, +7q34qter, } \\
-10 q 11.1 q 21.1,+10 q 26.2 q t e r, \\
-13 q 14.1 q 31,-14 q 12 q 22,+16 p,+17 q 24 q t e r, \\
+19 q,+22\end{array}$ \\
\hline A.W. & $\mathrm{pGBM}$ & $75 / \mathrm{m}$ & $\begin{array}{l}+3 q 24 q 27,+4 p,+5 p, \text { amp5p13.1p14, }+5 q 32, \\
+7, \text { amp7p12, }-9 p 21 \text { pter, }-10,-12 q 12 q 13.1, \\
-13 q 21.3 q 33,+14 q 23 q t e r,+15 q 21.1 q 24, \\
+17 p 11.2 p 12,+19,+20,+21 q 11.2 q 21\end{array}$ \\
\hline $\begin{array}{l}1934 / \\
11\end{array}$ & pGBM & $69 / f$ & $\begin{array}{l}+X,+3 q 24 q 26.1,+4 q 13.1 q 32,+6 q 15 q 22.3 \\
+7 q 21.1 q 32,-9 q 13 q 21.1,+11 q 14.1 q 22.3 \\
+12 q 21.3 q 24.1,-16 p,-17 p,-19 q 13.2 q t e r \\
-22\end{array}$ \\
\hline $\begin{array}{l}1381 / \\
06\end{array}$ & $\mathrm{pGBM}$ & $44 / m$ & $\begin{array}{l}-X,-Y,+1 q 32.2 q t e r,-2 p 15 p 23 \\
+2 q 11.1 q 21.3,-4 q 24 q 28,-6 q 21 q 22.3,+7 p \\
+7 q 33 q t e r,+8, \text { amp 8p23.1pter } \\
-10 q 22.1 q 25.1,-11 p,+11 q,-13 q 11 q 31 \\
-14 q 22 q 31,-21 q 11.1 q 22.1,-22\end{array}$ \\
\hline $\begin{array}{l}1080 / \\
10\end{array}$ & $\mathrm{pGBM}$ & $73 / \mathrm{m}$ & $\begin{array}{l}+X_{1}-1 p 34.1 \text { pter, }+4 p 14 p 16,+6 q 23.1 q 24, \\
+7 p,+8 p 22 \text { pter, }-9 q 34 q t e r,-10 \\
+12 p 11.2 p 13.1,+16 p 11.2 p 12,-16 q 24 q t e r \\
-17 q,+18 p 11.2 \text { pter }\end{array}$ \\
\hline
\end{tabular}


Table 3 Clinical chacteristics and CGH results of the Carmustin group (Continued)

\begin{tabular}{|c|c|c|c|}
\hline $\begin{array}{l}725 / \\
12\end{array}$ & pGBM & $50 / m$ & $\begin{array}{l}-X_{,}+1,+2,+3 p 13 p 25,+3 q 24,+4 p, \\
+4 q 26 q 34,+5 p 13.1 p 13.3,+5 q 14 q 15, \\
+5 q 23.1 q 34,-6 q,+7,+8 p 21.1 p t e r,+8 q 23, \\
-10 q 21.1 q 21.3,+11 q 14.1 q t e r,-13 q 33 q t e r, \\
-14 q 24.2 q t e r,+16 p,+16 q 11.2 q 22,+18,+19, \\
+20,+21\end{array}$ \\
\hline $\begin{array}{l}778 / \\
10\end{array}$ & pGBM & $70 / \mathrm{m}$ & $\begin{array}{l}+1 p 36.1,-4 q 34 q \text { ter, amp7p12, +7q11.1q22, } \\
\text {-10p15pter, +12q24.3qter, -13q23.1qter, } \\
+17 p 11.1 p 11.2,+18 p 11.1 p 11.2 \\
+19,+20 q 12 q 13.2\end{array}$ \\
\hline $\begin{array}{l}563 / \\
12\end{array}$ & $\mathrm{pGBM}$ & $60 / \mathrm{m}$ & -7q36qter \\
\hline $\begin{array}{l}904 / \\
11\end{array}$ & pGBM & $65 / m$ & $\begin{array}{l}-X_{,}+1 p 36.1 p 36.3,-4 p 16,-9 p 23 p t e r \\
+16 p 12 p 13.1\end{array}$ \\
\hline $\begin{array}{l}1346 / \\
06\end{array}$ & pGBM & $50 / f$ & $\begin{array}{l}-5 q 21 q 23.1,-8 q 23 q t e r,-10 q 23.2 q t e r,-15 \\
-17\end{array}$ \\
\hline $\begin{array}{l}175 / \\
12\end{array}$ & $\mathrm{pGBM}$ & $45 / m$ & $\begin{array}{l}-X_{1}+1 p 31.1 p 32.3,-2 p 11.2 p 13,+3 q 24 q 26.1 \\
+4 q 31.3 q 34,+7,+8 q 23 q 24.1,+16 q 12.2 q t e r \\
+16 p 12 p 13.2,-19 q,+21 q 11.2 q 22.1\end{array}$ \\
\hline
\end{tabular}

quantified by estimated hazard ratios (HR) estimates with corresponding 95\% confidence intervals.

\section{Results}

\section{Clinical data}

Overall median survival was 267 days $(95 \% \mathrm{CI}=[176$, 372]) with 309 days in group A $(95 \% \mathrm{CI}=[138,481])$ and 219 days in group B $(95 \% \mathrm{CI}=[151,372])$.

Univariate Cox models of clinical covariates revealed that age of onset of the disease had a statistically significant effect on OS (HR $=1.048,95 \% \mathrm{CI}=[1.024,1.072]$, $p<0.0001)$. The average age of onset was 60.1 years in group A and 60.2 years in group B. Gender did not show a significant effect on OS $(\mathrm{HR}=1.19,95 \% \mathrm{CI}=[0.715-$ 1.977], $p=0.504$ ) (see Table 2).

A statistically significant effect of additional treatment with carmustine wafer (group B) in comparison to the standard Stupp regime (group A) on OS could not be detected $(\mathrm{HR}=1.15,95 \% \mathrm{CI}=[0.708,1.888]$, $p=0.562$ ) [Fig. 2].

\section{Methylation analysis}

We found a MGMT methylation index (MI) of 58\% (21/ 36) in group A and a methylation index of $42 \%(15 / 36)$ in group B. At the p15 promotor we found a MI of $14 \%$ $(5 / 36)$ in group A and 25\% (9/36) in group B, respectively. The methylation index in p16 showed 8\% (3/36) in both groups.

Patients with an unmethylated MGMT showed a median OS of 6.6 months. If MGMT was methylated the median OS was 10.7 months. A univariate Cox model with MGMT as predictor results in MGMT has a statistically significant effect on OS $(\mathrm{HR}=0.593,95 \% \mathrm{CI}=$ $0.359-0.979, p=0.041)$. If stratified for treatment group there was neither in group A nor in group B a significant correlation between OS and MGMT methylation (group A: $p=0.0635$, group B: $p=0.319$ ) [Fig. 3a/b].

Subgroup analyses revealed that patients with a p15 methylation showed a significant shorter OS when administered to group B (median OS: 115 days) than in group A (median OS: 481 days) $(p=0.0332)$. A promotor methylation of p16 had no significant impact on any group [Fig. 4].

Within the whole population in this study $(N=72) 3$ patients showed an OS longer than 36 months, 2 of them were in group B, one in group A. All three cases showed a methylated MGMT promotor whereas p15 and p16 were not methylated.

\section{CGH}

In total, each tumor showed on average 11 aberrations and a total number of 754 aberrations could be detected. The distribution of alterations in both groups showed in general no differences [Fig. $5 \mathrm{a} / \mathrm{b}$ ]. We found different chromosomal alterations in all the analyzed tumor specimens. One of the most frequent alterations were gains on chromosome 7 in 85\% (61/72), chromosome 16 in 33\% (24/72), chromosome 4 in $22 \%(16 / 72)$, chromosome 5 in $21 \%(15 / 72)$, chromosome 12 in 19\% (14/72) and chromosome 20 in $22 \%(16 / 72)$.

In contrast losses were prevalently detected on the short arm of chromosome 9 in $47 \%$ (34/72), chromosome 10 in $67 \%$ (48/72), the long arm of chromosome 13 in 47\% (34/72), on chromosome 6 in 25\% (18/72), on the long arm of chromosome 22 in $22 \%(16 / 72)$, of the long arm of chromosome 4 in $19 \%(14 / 72)$ and on the long arm of chromosome 17 in 18\% (13/72) [Fig. 5 a/b].

We also found amplifications at $4 \mathrm{q} 12$ in $15 \%(11 / 72)$, at $7 \mathrm{p} 12$ in $19 \%(14 / 72)$ and in the region of $12 \mathrm{q}$ in $4 \%$ (3/72). In one case an amplification of $6 q 21$ and in another case of 8p23.1pter were found (Tables 3, 4 and 5).

Patients in group B whose tumors showed an amplification of 4q12 had a statistically significant reduced OS (log-rank test, $p=0.00835)$. An amplification of $4 \mathrm{q} 12$ for patients of group A did not show this worsening effect on OS. In contrast if a loss of chromosome 10 occurred in tumor samples, patients in group B, who additionally received carmustine wafer implantation, showed a significantly longer OS $(p=0.0123)$. This effect could not be observed in group A.

A loss of 13q in group B was significantly associated with a longer OS $(p=0.0364)$. Again, this effect could not be observed in group $\mathrm{A}$.

No further significant correlations regarding clinical, chromosomal and epigenetic data could be observed.

\section{Discussion}

The focus of this study was to find new molecular markers for treatment response in GBM. Only a few 
Table 4 Clinical characteristics and CGH results of the Controll group

\begin{tabular}{|c|c|c|c|}
\hline Cases & Histology & $\begin{array}{l}\text { Ages/ } \\
\text { gender }\end{array}$ & CGH-results \\
\hline $\begin{array}{l}1553 / \\
07\end{array}$ & pGBM & $82 / f$ & $\begin{array}{l}+2 q 12 q 33,+3 p 24.2 p 25,+3 q 22 q 24 \\
\text { amp4q12, }+7,-9 p 13 p 24,-10 \\
+11 q 13.2 q 14.1,-13 q 12.1 q 14.3,-15 q 21.3 q 23 \\
+16 p,+17 p 12 \text { pter, }+18 q 23 q \text { ter }\end{array}$ \\
\hline $\begin{array}{l}1042 / \\
12\end{array}$ & pGBM & $83 / f$ & $\begin{array}{l}-2 p 23 p 24,-4 p 12 p 15.1,-4 q 21.2 q 22, \\
\text { amp7p12, } \\
-16 q 13 q 21,-18 q 12.3 q 22\end{array}$ \\
\hline
\end{tabular}

$1117 / \mathrm{pGBM} \quad 71 / \mathrm{m} \quad+3,+7,-9 \mathrm{p} 21 \mathrm{pter},-10,-13,-14$

139/ pGBM 73/f

10

1588/ pGBM 70/

09

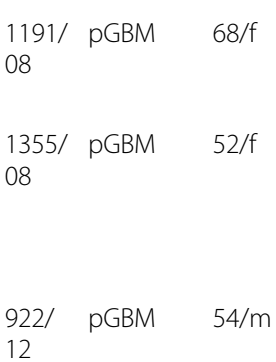

881/ $\mathrm{pGBM} \quad 40 / \mathrm{f}$

10

1229/ pGBM 72/m

08

375/ pGBM 72/m

10

1988/ pGBM

09

838/ sGBM 70/m

12

926/ pGBM 58/m

09

983/ pGBM 54/m -3p24.3pter, $-4 q 23 q 25,-6 q 16.3 q 21$

04
$+16 \mathrm{p} 11.2 \mathrm{p} 13$

$+1 p 13.1 p 33,+1 q 21.1 q 31,+2,+3 p 12 p 14.1$ $+3 p 25$ pter, $+3 q 13.1 q 21,+4 q 22 q 24,+5 p 14$ $+7,+8 p 23.1$ pter, $+8 q 11.2 q$ ter, $+9 p 23 p t e r$, $+9 \mathrm{q} 22.1 \mathrm{q} 33,-10,+13 \mathrm{q} 14.1 \mathrm{q} 21.3$,

$+14 q 12 q 21,-16 p 12 p 13.1,+16 q 23 q t e r$,

-17 p11.1p11.2, -18p11.2p11.3, -19, -22

$+\mathrm{X}_{,}+1 \mathrm{p} 21 \mathrm{p} 36.1,+3 \mathrm{p} 14.1 \mathrm{p} 24.1$

$-3 q 11.1 q 26.1,+5 q 23.2 q 32,+7$, amp $7 p 12$,

-9p22pter, $-10,+12 q 21.3 q 24.3$

$-17 p 11.1 p 11.2,+17 q 21.3 q 22$,

$-18 \mathrm{p} 11.1 \mathrm{p} 11.3,+19,+20$

+1p36.1pter, -1q42.2q43, amp4q12, +7,

$-10,-13 q 14.1 q 21.3,+16 q 11.2 q 21$,

$-17 q 12 q$ ter, $+18 p 11.1 p 11.2$

$-X,-1 \mathrm{q} 31,+6 \mathrm{q} 24 \mathrm{q} 26,+7,-8 \mathrm{p} 21.1 \mathrm{pter}$

-9p22pter, $-10,+11$ p15.1p15.4,

$+12 q 21.3 q$ ter, $-13 q 12.2 q$ ter, $+15 q 11.1 q 15$,

$+16,-18,-19 q 13.2 q$ ter, $+20 p,+20 q 11.1 q 12$,

$+21,-22$ q13.2qter

+1p13.1p13.3, +1q43qter, +2q12q14.1, -

2q37.1qter, +4p13pter, +4q24q25, +5, +7, $+8 p 12$ pter, +8q12q13, -10, +11p15.1pter, +11q13.1q23.3, -13q12.1q21.3, +16p12p13.1, +17p11.1p12, +19q13.1q13.2, +22

$+1,+2 p 21$ pter, $-5 q 14 q$ ter, $-6 q 14 q 21$,

$-9 \mathrm{p} 24 \mathrm{pter},-9 \mathrm{q} 31 \mathrm{q} 33,-10,-11 \mathrm{p}$,

$-12 q 12 q 13.1,-12 q 24.1 q 24.3,-13 q 14.1 q 21.2$,

$+16 \mathrm{p} 11.2 \mathrm{p} 13.1,+17 \mathrm{q} 21.1 \mathrm{qter}$

$+22 q 11.2 q 12.3$

$-1 p 31.2 p 36.1,-1 q 23 q 24,+3 q 26.1 q t e r$ amp4q12, +4q11q21.2, -4q33qter,

$-6 q 13 q 21,+7,-9 p 23 p 24,-10$,

$-11 q 14.1$ ter, $-13 q 14.1 q t e r,-14 q 22 q 32.1$

$+16,+17 p 11.1 p 12,-22 q 13.1 q 13.2$

$+1 p 36.1$ pter, $+2 p 21$ pter, $-4 p 11 p 12$

+6p12p21.3, -9p13p21, -13q21.1q31,

$+15 q 13 q 21.3,+17,+20 q 12 q$ ter

68/m amp7p12, $-10 p,+11 q 13.5 q 23.3,-12 p$,

$+20 q 31.1$ qter, $+22 q 12.3 q 13.1$

$+7,-9 p,-10,+19$

-3q27qter, -6q26qter, +7, amp7p12,

$-8 p 23.1$ pter, $-9 p 13 p t e r,-10,-16 p$

$+16 q 11.2 q 21,-18 q 21.3 q$ ter, +19 ,

$+20 q 12 q 13.2,-22 q 12.3 q$ ter

$-8 \mathrm{q} 24.1 \mathrm{qter},-10,-11 \mathrm{q} 23.3 \mathrm{qter}$,

$-13 q 22 q t e r,-17 q 25 q t e r$
Table 4 Clinical characteristics and CGH results of the Controll group (Continued)

\begin{tabular}{|c|c|c|c|}
\hline $\begin{array}{l}837 / \\
12\end{array}$ & pGBM & $54 / f$ & $\begin{array}{l}+7,-8 p 22 \text { pter, }-9 p 13 p 24,-10 \\
+17 q 21.2 q 22,+18 q 12.1 q 12.3,+19 p\end{array}$ \\
\hline $\begin{array}{l}1078 / \\
09\end{array}$ & $\mathrm{pGBM}$ & $54 / \mathrm{m}$ & $\begin{array}{l}+ \text { Xp, }+ \text { Xq11.1q21.3, +1, -3p21.3pter, } \\
+3 q 13.2 q \text { ter, }-4 p 14 p t e r,+4 q 28 q 34,+5 p \\
+5 q 11.2 q 13.2,-5 q 33.1 q t e r,+6 p,+6 q 21 q t e r \\
+7,+8,-9 p 23 p t e r,+12 p,+12 q 11 q 13.1,-13 \\
-14 q 21 q t e r, \text { amp 14q11.2q12, } \\
-20,+217,+19\end{array}$ \\
\hline $\begin{array}{l}776 / \\
05\end{array}$ & $\mathrm{pGBM}$ & $50 / m$ & $+3 p 23 p 24.3,+5 q 23.3 q 31.1,+7 p 14 p 22$ \\
\hline $\begin{array}{l}1861 / \\
03\end{array}$ & pGBM & $43 / \mathrm{m}$ & $\begin{array}{l}+2 p 23 p 24,+2 q 22 q 34,+6 q 22.3 q 24,+7,-8 p \\
-9 p 23 p t e r,-10, \text { amp12q13.12q21.3, +13, } \\
-15 q 12 q 14,+18 q 12.1 q 21.2,-22 q 11.2 q 13.1\end{array}$ \\
\hline $\begin{array}{l}1216 / \\
11\end{array}$ & pGBM & $50 / m$ & $\begin{array}{l}\text { +7p11.1pter, amp7p12, -9p21 pter, } \\
-10 q 21 q \text { ter, +11q21q22.3, +12q13.1qter, } \\
-13 q 14.1 q 21.3,-22 q 13.3 q \text { ter }\end{array}$ \\
\hline $\begin{array}{l}1110 / \\
05\end{array}$ & pGBM & $41 / m$ & $+7 p$ \\
\hline $\begin{array}{l}1457 / \\
03\end{array}$ & pGBM & $37 / \mathrm{m}$ & $\begin{array}{l}-5 q 34 q t e r,+8 q 21.3 q \text { ter, +10p1 1.2pter, } \\
-12 q 24.1 q 24.3,-13 q 33 q t e r,+18 q 12.2 q 21.1\end{array}$ \\
\hline $\begin{array}{l}378 / \\
12\end{array}$ & pGBM & $59 / f$ & $\begin{array}{l}\text { +1q42.2qter, }-4 p 11 \text { p13, -4q11q13.1, } \\
+5 p 14 p 15.2,-6 q,+7, \text { amp7p12, +8p22pter, } \\
\text {-9p13pter, }-10,-11 p 12 p 14,-13 q 14.3 q 33, \\
+14 q 11.1 q 13,-16 q 23 q 24,-17 p 13 p t e r \\
-18 p 11.1 p 11.3,+19 q,+20,+21 q 11.1 q 22.1\end{array}$ \\
\hline $\begin{array}{l}1583 / \\
08\end{array}$ & pGBM & $68 / f$ & $\begin{array}{l}-2 p 23 p 24,-5 q 21 q 31.1,+5 q 33.1 q t e r \\
-6 q 12 q 14,+7,+8 p 23.1 p t e r,-8 p 11.1 p 12, \\
\text {-9q22.3qter, }-10,-13 q 33 q t e r \\
+16 p 11.2 p 13.1,+17 p 11.1 p 12, \\
+19 p 13.1 p 13.3,+20,+21 q 11.2 q 22.1 \\
-22 q 11.2 q t e r\end{array}$ \\
\hline $\begin{array}{l}655 / \\
09\end{array}$ & pGBM & $71 / f$ & $\begin{array}{l}-6 q 12 q \text { ter },+7,-9 p 21 \text { pter, }-10 \\
-12 q 21.3 q 23,-13 q 12.3 q \text { ter, }+20\end{array}$ \\
\hline $\begin{array}{l}291 / \\
09\end{array}$ & pGBM & $51 / f$ & $\begin{array}{l}+4 p 14,-6 q 26 q t e r,+7 p 13 p t e r \\
+18 q 11.1 q 21.1,+ \text { Xq21.2q27 }\end{array}$ \\
\hline $\begin{array}{l}156 / \\
12\end{array}$ & sGBM & $76 / f$ & $\begin{array}{l}-1 \text { p34.2pter, }+5,+7,+9 q 13 q \text { ter, } \\
-15 q 21.3 q \text { ter, },-16 q 11.2 q 13,-17 q 11.2 q 21.1\end{array}$ \\
\hline $\begin{array}{l}1286 / \\
08\end{array}$ & pGBM & $65 / m$ & $+7,-10 \mathrm{q} 25.1 \mathrm{qter},+16 \mathrm{p} 12$ \\
\hline $\begin{array}{l}1458 / \\
10\end{array}$ & $\mathrm{pGBM}$ & $49 / f$ & $+7 q 11.2,-13 q 12.3 q 14.1,-15 q 26.1$ qter \\
\hline $\begin{array}{l}1431 / \\
05\end{array}$ & $\mathrm{pGBM}$ & $69 / f$ & $\begin{array}{l}-2 p 12 p 15,-2 q 37.1 \text { qter, }-3 p 24.1 \text { pter, } \\
\text { amp4q12, -5p15.3pter, }+7,-9 p 13 p 23, \\
-9 q 33 q t e r,-10,-11 p 12 p 15.3,-12 q 24.1 \text { qter, } \\
-13 q 13 q 21.1,-16 q 23 q 24\end{array}$ \\
\hline $\begin{array}{l}T \\
6929\end{array}$ & $\mathrm{pGBM}$ & $50 / \mathrm{m}$ & $\begin{array}{l}+1 p 21 p 31.2,+3 q 12 q 13.2,+3 q 25.3 q \text { ter } \\
-4 p 16 p t e r,+6 q 12 q 13,+7,-9 p 21 p 23 \\
\text {-9q13q23.1, -10,-11q23.1q23.3, } \\
-12 q 22 q t e r,-13 q 12.3 q t e r,-17 \\
-18 p 11.1 p 11.3,-20,+ \text { Xq13q23 }\end{array}$ \\
\hline $\begin{array}{l}338 / \\
12\end{array}$ & pGBM & $72 / \mathrm{m}$ & $\begin{array}{l}-X_{1}+7,-9 p 21 \text { pter, }-11 q 12 q 13.2 \\
+17 p 11.2 p 12,+20\end{array}$ \\
\hline $\begin{array}{l}791 / \\
08\end{array}$ & pGBM & $56 / \mathrm{m}$ & No aberrrations \\
\hline $\begin{array}{l}663 / \\
12\end{array}$ & $\mathrm{pGBM}$ & $65 / f$ & $\begin{array}{l}+4,+7,-10 p 14 \text { pter, }-10 \mathrm{q} 25.2 \mathrm{qter}, \\
+12 \mathrm{q} 13.1 \mathrm{q} 13.3,-15\end{array}$ \\
\hline $\begin{array}{l}754 / \\
12\end{array}$ & pGBM & $56 / \mathrm{m}$ & $\begin{array}{l}+5 p,+5 q 11.2 q 14,+7,-10,-13 q 11 q 21.1 \\
-17 q 24 q \text { ter, }-19 q\end{array}$ \\
\hline
\end{tabular}


Table 4 Clinical characteristics and CGH results of the Controll group (Continued)

\begin{tabular}{|c|c|c|}
\hline $\begin{array}{l}2512 / \text { pGBM } \\
11\end{array}$ & $42 / m$ & $\begin{array}{l}-X p,+4 q, \text { amp4q12, }-5 q 13.3 q \text { ter, }-7 p 14 p t e r, \\
+7 q,+8 p,-9 q 34.1 q \text { ter, }-10 q 22.3 q t e r,-11 p, \\
-11 q 24 q \text { ter, }-12 p,-12 q 14 q 23,-13, \\
-18 p 11.2 \text { pter, }-18 q 22 q \text { ter, }-21\end{array}$ \\
\hline
\end{tabular}

previous retrospective and prospective studies have analyzed the combination of carmustine wafer implantation with the combined standard chemoradiation protocol for the treatment of newly diagnosed glioblastoma [26-35].

We report here the impact of carmustin wafer implantation together with the combined standard chemoradiation protocol in newly diagnosed supratentorial glioblastoma in adults. To overcome the limitations inherent to retrospective observational studies, we performed a confirmatory case matched analysis $(N=72)$. The data from our study confirmed previous trials suggesting that MGMT is a predictive marker for TMZ therapy response $[11,14-19]$. We also found a significant correlation between MGMT methylation status and OS in our total collective. This significance vanished when the patients were stratified for treatment group A or B. This may result from the limited number per treatment cohort. Losses of chromosome 10 are among the most frequent in GBM [36-38]. Patients with additional carmustine wafer treatment and a loss of chromosome 10 showed a significantly longer OS than patients without that chromosomal loss. An explanation for this could be the MGMT gene locus on 10q21. A loss of this region results in a loss of MGMT expression and therefore ameliorate the treatment response of both TMZ and the local carmustin therapy. Wemmert et al. could show a similar effect regarding TMZ therapy alone [38]. In our trial this effect occurred only in patients receiving both TMZ/RT $\rightarrow$ TMZ regime and carmustin wafer implantation, but not in patients who received TMZ/RT $\rightarrow$

Table 5 Overview of the chromosomal alterations

\begin{tabular}{lll}
\hline Alteration & $\begin{array}{l}\text { Group B } \\
\text { frequency }\end{array}$ & $\begin{array}{l}\text { Group A } \\
\text { frequency }\end{array}$ \\
\hline amplification 4q12 & $6 / 36(17 \%)$ & $5 / 36(14 \%)$ \\
gain on chromosome 7 & $30 / 36(83 \%)$ & $31 / 36(86 \%)$ \\
amplification on 7p12 & $8 / 36(22 \%)$ & $6 / 36(17 \%)$ \\
loss on 9p & $16 / 36(44 \%)$ & $18 / 36(50 \%)$ \\
loss on 10q & $23 / 36(64 \%)$ & $25 / 36(69 \%)$ \\
gain on 12q & $9 / 36(25 \%)$ & $6 / 36(17 \%)$ \\
loss of chromosome 13 & $13 / 36(36 \%)$ & $21 / 36(58 \%)$ \\
loss of chromosome 20 & $8 / 36(22 \%)$ & $9 / 36(25 \%)$ \\
loss of chromosome 22 & $8 / 36(22 \%)$ & $7 / 36(19 \%)$ \\
\hline
\end{tabular}

TMZ regime alone. Possibly other so far unknown gene loci might also play an important role.

Mutations and deletions of p15 and p16 are frequent genetic alterations in glial tumors [39-43]. p15 and p16 inhibits CDK4 and CDK6, therefore p15 and p16 act as tumor suppressors and lead to cell cycle arrest in the late G1 phase [33]. Previous studies indicated that a loss of expression, resulting from deletion, mutations or methylation of p15 and p16 is associated with a significantly worse prognosis for survival in glioblastoma [21, $38,44,45]$. Our data supports this point of view, at least regarding $\mathrm{p} 15$. Interestingly patients administered to group B with methylated p15 showed significantly the shortest OS of all subgroups within our trial. The low number of only six p16 methylated tumors shows that p16 is not of significant impact on our collective. Considering the findings of the literature this is not surprising [21, 38, 44-46].

Another important finding of our study is the amplification of the region $4 \mathrm{q} 12$ as a prognostic marker in patients additionally treated with carmustine wafer. Patients in group B whose tumor showed this amplification had a significantly shortened OS. PDGFR $\alpha$, a tyrosine kinase, is located in the region of $4 \mathrm{q} 12$. PDGFR $\alpha$ is known to play a major role in tumor angiogenesis by stimulation of cell growth $[47,48]$. The exact mechanism of PDGFR $\alpha$ in vasculogenesis and tumor angiogenesis is yet unknown, but an overexpression of PDGFR $\alpha$ caused by gene amplification may result in more aggressive tumor growth.

This effect was not observable in group A. Maybe PDGFR $\alpha$ is not the only determinant gene influencing OS and due to the described tumor heterogeneity in GBM it did not reach a statistical significant level [24, 49, 50].

We also detected a better prognosis in group $B$ if chromosome 13 or parts of chromosome 13 were lost. This is concordant with previous findings where a survival benefit in patients treated with alkylating agents was found, if chromosome 13 was lost [38]. A further genetic hotspot is the RB1 gene, which is located on 13q14.2. Maybe a loss of this gene influences the oncological behavior of tumor cells in such a manner that additional carmustine wafer therapy shows an improved impact on the clinical course. This effect could not be shown in the standard therapy group treated with Stupp regime. Hence a loss of chromosome 13 is maybe a prognostic marker for an ameliorated clinical course which would recommend the implantation of carmustine wafers.

Besides to the molecular findings described above, we found no significant survival benefit between group A and $\mathrm{B}$ in general. We think it is not recommendable to use additional carmustine wafer implantation in every single case. This is conclusive with the data of other clinical 
studies and the widespread use of carmustine wafers is highly controversial due to its debatable clinical impact. Pallud et al. could e.g., not show a long-term benefit regarding overall survival (OS) in a cohort of 354 patients [9]. This controversy is even more understandable if the clinical side effects of carmustine wafer implantation is taken into account. Especially operative wound infection and cerebral edema can be increased [10].

Therapy with carmustine wafers should be individually assessed for each patient. This also represents the current opinion in treatment guidelines, in general.

Overall, our findings suggest that carmustin wafer implantation in combination with maximal safe resection, followed by combined standard chemoradiation protocols, is a promising treatment option for patients with supratentorial glioblastoma harboring MGMT promoter methylation.

\section{Conclusion}

A clinical benefit for the widespread use of additional carmustine wafer implantation could not be found. However, carmustine wafer implantation shows a significantly improved overall survival if chromosome 10 and particularly 10q or chromosome13 are deleted. In cases of 4q12 amplification and in cases of a methylated p15 promotor, the use of carmustine wafers is especially not recommended.

The MGMT promoter methylation is a strong prognostic Biomarker for benefit from temozolomide and BCNU chemotherapy.

Therefore we propose to use BCNU wafers in a second line therapy, when the chromosomal and epigenetic data from the primary tumor are available. However, owing to the small number of patients these findings would need to be corroborated in lager patients cohorts.

\section{Abbreviations}

CGH: Comparative genomic hybridization; TMZ: Temozolomide; WHO: World Health Organization

\section{Acknowledgments}

We thank Cornelia Lerner, Silke Wemmert and Ulrike Bechtel for expert technical assistance and helpful discussions.

\section{Funding}

This work was supported by Archimedes GmbH (L204150209).

\section{Availability of data and materials}

Please contact author for data requests.

\section{Authors' contributions}

US carried out the comparative genetic hybridization and wrote the manuscript, SC and EJ carried out the methylation analysis, KK performed the statistical analysis, OJ and RK did surgery of the patients and participated in its design. All authors read and approved the final manuscript.

\section{Competing interests}

Ketter R. had done speaking engagements (including travel and accommodation) and is consultant for Kyowa Kirin $\mathrm{GmbH}$.

All other authors declare they have no competing interests.
Consent for publication

Not applicable

\section{Ethics approval and consent to participate}

Written informed consent was obtained from each patient participating in the study. We have a positive vote of the Ethics committee of the Saarland University (Ethik-Nr. 93-16).

\section{Publisher's Note}

Springer Nature remains neutral with regard to jurisdictional claims in published maps and institutional affiliations.

\section{Author details \\ 'Department of Neurosurgery, Saarland University, 66421 Homburg/Saar, Germany. ${ }^{2}$ Division of Biostatistics and Bioinformatics, Department of Oncology, Sidney Kimmel Comprehensive Cancer Center, Johns Hopkins University School of Medicine, Baltimore, MD, USA.}

Received: 21 March 2017 Accepted: 24 April 2017

Published online: 04 May 2017

References

1. Louis DN. Molecular pathology of malignant gliomas. Annu Rev Pathol. 2006;1:97-117.

2. Dolecek TA, Propp JM, Stroup NE, Kruchko C. CBTRUS statistical report: primary brain and central nervous system tumors diagnosed in the United States in 2005-2009. Neuro-Oncology. 2012;14 Suppl 5:1-49.

3. Wen PY, Kesari S. Malignant gliomas in adults. N Engl J Med. 2008:359(5):492-507.

4. Clarke J, Butowski N, Chang S. Recent advances in therapy for glioblastoma. Arch Neurol. 2010:67(3):279-83.

5. Stupp R, Mason WP, van den Bent MJ, Weller M, Fisher B, Taphoorn MJ, et al. Radiotherapy plus concomitant and adjuvant temozolomide for glioblastoma. N Engl J Med. 2005;352:987-96.

6. WU MP, Tamada JA, Brem H, Langer R. In vitro degradation of controlled release polymers for intracranial surgical therapy. J Biomed Mater Res. 1994;28(3):387-95

7. De Bonis P, Anile C, Pompucci A, et al. Safety and efficacy of Gliadel wafers for newly diagnosed and recurrent glioblastoma. Acta Neurochir. 2012;154:1371-8.

8. Westphal M, Hilt DC, Bortey E, Delavault P, Olivares R, Warnke PC, Whittle IR, Jääskeläinen J, Ram Z. A phase 3 trial of local chemotherapy with biodegradable carmustine (BCNU) wafers (Gliadel wafers) in patients with primary malignant glioma. Neuro-Oncology. 2003;4:79-88.

9. Pallud J, Audureau E, Noel G, Corns R, Lechapt-Zalcman E, et al. Long-term results of carmustine wafer implantation for newly diagnosed glioblastomas: a controlled propensity-matched analysis of a French multicenter cohort. Neuro-Oncology. 2015;17(12):1609-19.

10. Weber EL, Goebel EA. Cerebral edema associated with gliadel wafers: two case studies. Neuro-Oncology. 2005;7:84-9.

11. Hegi ME, Liu L, Herman JG, et al. Correlation of O6-methylguanine methyltransferase (MGMT) promoter methylation with clinical outcomes in glioblastoma and clinical strategies to modulate MGMT activity. J Clin Oncol. 2008;26(25):4189-99.

12. Hegi ME, Diserens AC, Gorlia T, et al. MGMT gene silencing and benefit from temozolomide in glioblastoma. N Engl J Med. 2005;352(10):997-1003.

13. Zhang W, Zhang J, Hoadley K, Kushwaha D, Ramakrishnan V, et al. miR181d: a predictive glioblastoma biomarker that downregulates MGMT expression. Neuro-Oncology. 2012;14(6):712-9.

14. Weller M, Stupp R, Reifenberger G, Brandes AA, van den Bent MJ, Wick W, et al. MGMT promotor methylation in malignant gliomas; ready for personalized medicine? Nat Rev Neurol. 2010;6:39-51.

15. Wick W, Hartmann C, Engel C, Stoffels M, Felsberg J, Stockhammer F, et al. NOA-04 randomized phase III trial of sequential radiochemotherapy of anaplastic glioma with procarbazine, lomustine, and vincristine or temozolomide. J Clin Oncol. 2009;27:5874-80.

16. van den Bent MJ, Dubbink HJ, Sanson M, van der Lee-Haarloo CR, Hegi M, Jeuken JW, et al. MGMT promotor methylation is prognostic but not predictive for outcome to adjuvant PCV chemotherapy in anaplastic oligodendroglial tumours: a report from EORTC Brain Tumour Group Study 26951. J Clin Oncol. 2009;27:5881-6.

17. Bady P, Sciuscio D, Diserens AC, Bloch J, van den Bent MJ, Marosi C, et al. MGMT methylation analysis of glioblastoma on the Infinium 
methylationBeadChip identifies two distinct $\mathrm{CpG}$ regions associated with gene silencing and outcome, yielding a prediction model for comparison across datasets, tumor grades, and CIMP status. Acta Neuropathol. 2012;124:547-60.

18. Wick W, Meisner C, Hentschel B, Platten M, Schilling A, Wiestler B, et al. Prognostic or predictive value of MGMT promotor methylation in gliomas depends on IDH1 mutation. Neurology. 2013;81:1515-22.

19. Tolcher AW, Gerson SL, Denis I, Geyer C, Hammond LA, Patnaik A, et al. Marked inactivation of O6-alkylguanine-DNA alkyltransferase activity with protracted temozolomide schedules. Br J Cancer. 2003;88:1004-11.

20. Wait SD, Prabhu RS, Burri SH, Atkins TG, Asher AL. Polymeric drug delivery for the treatment of glioblastoma. Neuro-Oncology. 2015;7(S2):9-23.

21. Wemmert S, Bettscheider M, Alt S, Ketter R, Kammers K, Feiden W, et al. p15 promoter methylation - a novel prognostic marker in glioblastoma patients. Int J Oncol. 2009;34(6):1743-8.

22. Tada K, Shiraishi S, Kamiryo T, Nakamura H, Hirano H, Kuratsu J, et al. Analysis of loss of heterozygosity on chromosome 10 in patients with malignant astrocytic tumors: correlation with patient age and survival. J Neurosurg. 2001;95(4):651-9.

23. Herman JG, Graff JR, Myohanen S, et al. Methylation-specific PCR: a novel PCR assay for methylation status of CpG islands. Proc Natl Acad Sci U S A. 1996;93: 9821-6.

24. Jung V, Romeike BFM, Henn W, Feiden W, Moringlane JR, Zang KD, Urbschat SM. Heterogeneity of gliomas revealed by microdissection dop-pcr and comparative genomic hybridization. J Neuropathol Exp Neurol. 1999;58:993-9.

25. Cox DR. Regression models and life tables (with discussion). JR Stat Soc B. 1972;34(2):187-220

26. Mcgirt MJ, Than KD, Weingart JD, et al. Gliadel (BCNU) wafer plus concomitant temozolomide therapy after primary re of glioblastoma multiforme. J Neurosurg. 2009;110(3):583-8.

27. Duntze J, Litré CF, Eap C, et al. Implanted carmustin wafers followed by concomitant radiochemotherapy to treat newly diagnosed malignant gliomas; prospective, observational. Multicentre study on 92 cases. Ann Surg Oncol. 2012;20(6):2065-72.

28. Affronti ML, Heery CR, Herndon JE, et al. Overall survival of newly diagnosed glioblastoma patients receiving carmustine wafers followed by radiation and concurrent temozolomide plus rotational multiagent chemotherapy. Cancer. 2009;115(15):3501-11.

29. Menei $P$, Metellus $P$, Parot-Schinkel E, et al. Biodegradable carmustin wafers (Gliadel) alone or in combination with chemoradiotherapy: the French experience. Ann Surg Oncol. 2010;17(7):1740-6.

30. La Rocca RV, Mehdorn HM. Localized BCNU chemotherapy and the multimodal management of malignant glioma. Curr Med Res Opin. 2009;25(1):149-60.

31. Pan E, Mitchell SB, Tsai JS. A retrospective study of the safety of BCNU wafers with concurrent temozolomide and radiotherapy and adjuvant temozolomide for newly diagnosed glioblastoma patients. J Neuroonkol. 2008;88(3):353-7.

32. Pavlov V, Page P, Abi-Lahoud $G$ et al. Combining intraoperative carmustine wafers and Stupp regimen in multimodal first-line treatment of primary glioblastomas. Br J Neurosurgery. 2015. doi: 10.3109/02688697.2015.1012051

33. Bock HC, Puchner MJA, Lohmann F, et al. First-line treatment of malignant glioma with carmustine implants followed by concomitant radiochemotherapy: a multicentre experience. Neurosurg Rev. 2010;33(4):441-9.

34. Noel $G$, Schott R, Froelich $S$, et al. Retrospective comparison of chemoradiotherapy followed by adjuvant chemotherapy, with or without prior gliadel implantation (carmustine) after initial surgery in patients with newly diagnosed high-grade gliomas. Int J Radiat Oncol Biol Phys. 2012;82(2):749-55.

35. Miglierini $\mathrm{P}$, Bouchekoua $\mathrm{M}$, Rousseau $\mathrm{B}$, et al. Impact of the per-operatory application of GLIADEL wafers (BCNU, carmustine) in combination with temozolomide and radiotherapy in patients with glioblastoma multiforme: efficacy and toxicity. Clin Neurol Neurosurg. 2012;114(9):1222-5.

36. Karlbom AE, James CD, Boethius J, Cavenee WK, Collins VP, Nordenskjöld M, Larsson C. Loss of heterozygosity in malignant gliomas involves at least three distinct regions on chromosome 10. Human genetics. 1993;92(2):169-74.

37. Kimmelman AC, Ross DA, Liang BC. Loss of heterozygosity of chromosome 10p in human gliomas. Genomics. 1996;34(2):250-4. doi:10.1006/geno.1996.0277.

38. Wemmert S, Ketter R, Rahnenführer J, Beerenwinkel N, Strowitzki M, Feiden $W$, et al. Patients with high-grade gliomas harboring deletions of chromosomes $9 p$ and $10 q$ benefit from temozolomide treatment. Neoplasia. 2005;7(10):883-93.

39. Jen J, Harper JW, Bigner SH, et al. Deletion of p16 and p15 genes in brain tumors. Cancer Res. 1994;54:6353-8.
40. Schmidt EE, Ichimura K, Reifenberger G, Collins VP. CDKN2 (p16/MTS1) gene deletion or CDK4 amplification occurs in the majority of glioblastomas. Cancer Res. 1994;54:6321-4.

41. Simon M, Köster G, Menon AG, Schramm J. Functional evidence for a role of combined CDKN2A [p16/p14(arf)]/CDKN2B (p15) gene inactivation in malignant gliomas. Acta Neuropathol. 1999;98:444-52.

42. Rasheed A, Herndon JE, Stenzel TT, et al. Molecular markers of prognosis in astrocytic tumors. Cancer. 2002;94:2688-97.

43. Wemmert S, Romeike BF, Ketter R, Steudel WI, Zang KD, Urbschat S. Intratumoral genetic heterogeneity in pilocytic astrocytomas revealed by CGH-analysis of microdissected tumor cells and FISH on tumor tissue sections. Int J Oncol. 2006;28(2):353-60.

44. Gil J, Peters G. Regulation of the INK4b-ARF-INK4a tumour suppressor locus: all for one or one for all. Nat Rev Mol Cell Biol. 2006;7:667-77.

45. Kamiryo T, Tada K, Shiraishi S, et al. Analysis of homo- zygous deletion of the p16 gene and correlation with survival in patients with glioblastoma multiforme. J Neurosurg. 2002;96:815-22.

46. Wong $\mathrm{IH}$, Lo YM, Yeo W, Lau WY, Johnson PJ. Frequent p15 promoter methylation in tumor and peripheral blood from hepatocellular carcinoma patients. Clin Cancer Res. 2000;6(9):3516-21.

47. Shih AH, Holland EC. Platelet-derived growth factor (PDGF) and glial tumorigenesis. Cancer Lett. 2006;232(2):139-47. doi:10.1016/j.canlet.2005.02.002.

48. Varela M, Ranuncolo SM, Morand A, Lastiri J, De Kier J, Elisa B, Puricelli LI, Pallotta MG. EGF-R and PDGF-R, but not bcl-2, overexpression predict overall survival in patients with low-grade astrocytomas. J Surg Oncol. 2004; 86(1):34-40. doi:10.1002/jso.20036.

49. Cui XL, Zhao ZG, Ren XH, Sui DL, Chu JS, Tang K, et al. Characteristics of combining loss of heterozygosity of 1p/19q in glioma. Zhonghua wai ke za zhi [Chinese journal of surgery]. 2010;48(11):852-5.

50. Cairncross JG, Ueki K, Zlatescu MC, Lisle DK, Finkelstein DM, Hammond RR, et al. Specific genetic predictors of chemotherapeutic response and survival in patients with anaplastic oligodendrogliomas. J Natl Cancer Inst. 1998; 90(19):1473-9

\section{Submit your next manuscript to BioMed Central and we will help you at every step:}

- We accept pre-submission inquiries

- Our selector tool helps you to find the most relevant journal

- We provide round the clock customer support

- Convenient online submission

- Thorough peer review

- Inclusion in PubMed and all major indexing services

- Maximum visibility for your research

Submit your manuscript at www.biomedcentral.com/submit
( Biomed Central 